\title{
ACESSO AO TRABALHO INCLUSIVO PARA PESSOAS COM DEFICIÊNCIA: UMA SISTEMATIZAÇÃO DE BOAS PRÁTICAS BASEADA EM LEVANTAMENTO SISTEMÁTICO DA LITERATURA
}

\author{
Tatiana Ferreira da Costa e Silva ${ }^{1} 1$ e Fernando Oliveira de Araujo iD2
}

\section{Resumo}

A inclusão das pessoas com deficiência (PCD) nos espaços públicos e privados é um fator relevante a ser tratado nos dias atuais. Mas isso não deve ser entendido como um problema de assistencialismo, e, sim, como uma forma de pertencimento dessa classe, que passa a provar, constantemente, o quanto pode ser produtiva e contributiva para as organizações. Dessa forma, o presente artigo tem a finalidade de apontar um conjunto de boas práticas observadas em organizações públicas e privadas, com base em levantamento sistemático da literatura. Para apoiar esse objetivo, utilizou-se como metodologia o protocolo Cochrane, que suporta levantamentos sistemáticos na área da saúde. Para tanto, foram realizadas análises sistemáticas em três bases internacionais de periódicos, além de uma base nacional de teses e dissertações. Foi possível achar como resultados a prática da promoção da diversidade por meio da inclusão, via mercado de trabalho; o respeito às diferenças, a sensação de pertencimento das PCD, o aprendizado e a construção de competências entre os trabalhadores com deficiência. O artigo oferece uma contribuição para os diversos setores que empregam pessoas com deficiência, ajudando-os a gerenciarem e empregarem ações que incluam e empoderem, de forma efetiva, seus empregados com necessidades especiais.

Palavras-chave: Pessoas com Deficiência; Inclusão Social; Mercado de Trabalho; Empoderamento.

\section{ACCESS TO INCLUSIVE WORK FOR PEOPLE WITH DISABILITIES: A SYSTEMATIZATION OF GOOD PRACTICES BASED ON SYSTEMATIC LITERATURE SURVEY}

\section{Abstract}

The inclusion of people with disabilities (PwD) in public and private spaces is a relevant factor to be addressed today. But this should not be understood as a problem of assistance, but as a form of belonging to this class, which is constantly proving how productive and contributory it can be for organizations. Thus, this article aims to systematize a set of good practices observed in organizations based on a systematic literature survey. To support this objective, the Cochrane protocol was used as a methodology, which supports systematic surveys in the health area. For that, systematic analyzes were carried out in three international bases of journals, in addition to a national base of theses and dissertations. It was possible to find as

${ }^{1}$ Mestranda em Sistemas de Gestão pela Universidade Federal Fluminense (UFF). Assistente em Administração da UFF.

${ }^{2}$ Pós-Doutorado em Engenharia de Produção pela Universidade Federal do Rio Grande do Sul (UFRGS). Professor, pesquisador e extensionista do Departamento de Engenharia de Produção da Universidade Federal Fluminense (UFF). 
results the practice of promoting diversity through inclusion, via the labor market; respect for differences, the feeling of belonging to the PWD, learning and building skills among workers with disabilities. The article offers a contribution to the various sectors that employ people with disabilities, helping them to manage and employ actions that effectively include and empower their employees with special needs.

Keywords: People with Disabilities; Social Inclusion; Job Market; Empowerment.

\section{Introdução}

A inclusão social é uma premissa essencial para o aperfeiçoamento da democracia que respeita as particularidades dos cidadãos ao reconhecer as suas diferenças e, concomitantemente, fomenta o desenvolvimento de suas habilidades (COSTA; OLIVEIRA, 2017).

Em conformidade com Sassaki (2010), incluir as pessoas com deficiência nos ambientes públicos ou privados torna-se uma condição importante e contributiva para esses profissionais, como também para a sociedade, além de possuir um papel relevante sobre a ótica social e legal. Para que uma sociedade possa desenvolver a inclusão, primeiramente, é necessário acolher todos os indivíduos e respeitar as suas diferenças e particularidades, além das suas limitações. A discriminação ocorre pelo fato de uma determinada sociedade não ser educada para lidar com o que é diferente.

Em estudo realizado por Vornholt et al.(2018), os autores enfatizaram que no contexto internacional, especialmente em vários países europeus, viu-se a necessidade de reconhecer a integração das PCD, ao criar um mercado de trabalho para esses indivíduos de forma que pudessem incluí-los na sociedade.

Já em âmbito nacional, a Lei Brasileira de Inclusão da Pessoa com Deficiência (Estatuto da PCD) de no. 13.146/2015 visa reforçar o processo de inclusão das pessoas com deficiência no país, sendo um instrumento que tem a pretensão de assegurar e promover em condições de igualdade, o exercício dos direitos e das liberdades fundamentais das PCD, com a finalidade de potencializar a sua inclusão na sociedade e a sua cidadania.

No que concerne ao mercado de trabalho para as pessoas com deficiência, nota-se que, na iniciativa privada, a inclusão profissional das PCD é regida pela Lei 8.213/91 (art. 93). A referida normativa, também conhecida como Lei de Cotas, estabelece a obrigação para empresas com mais 100 empregados de preencher entre $2 \%$ e $5 \%$ de suas vagas para candidatos que possuam alguma deficiência (SOUZA; DIEGUES; CARVALHO, 2012).

Neves, Prais e Silveira (2015) relatam que as pessoas com deficiência são amparadas por essa lei, por meio de uma obrigatoriedade, e caso essas empresas não a cumpram, são penalizadas. Porém, isso não é o suficiente para que a inclusão aconteça amplamente. Nesse ínterim, a legislação não consegue sozinha suprir essa lacuna. A sociedade precisa ser conscientizada de que as PCD possuem reais limitações, mas também potenciais. Todos estão sujeitos a adquirir uma deficiência ou lidar com as PCD, dessa forma, a população precisa ser esclarecida de maneira abrangente.

No âmbito da coisa pública, de acordo com Costa e Oliveira (2017), a inserção de PCD no ambiente laboral dá-se por meio de concurso público, sendo o Decreto no 3.298/1999 uma das normas basilares, dispondo sobre a "Política Nacional para a 
Integração da Pessoa Portadora de Deficiência". Tal política pública assegura às PCD o direito de se inscreverem em concurso público, em igualdade de condições com os demais candidatos, para o provimento de cargos cujas atribuições sejam compatíveis com suas deficiências.

Ao abordar assuntos como deficiência, emprego e adaptações do local de trabalho, são sugeridas necessidades de criar estratégias eficazes que possam trazer garantias de acesso e permanência das pessoas com deficiência ao mercado de trabalho. Os resultados destas reflexões apontam que as pessoas com deficiência enfrentam grandes desafios quando reflete sobre a sua entrada e continuidade no ambiente laboral (VORNHOLT et al., 2018; KUZNETSOVA; BENTO, 2018).

Acrescente-se que, em virtude de preconceitos muito velados, segundo Vash (1988), empresas ainda resistem em contratar colaboradores que possuam alguma deficiência. Outros fatores como acreditarem que esses indivíduos não exercem suas funções com êxito ou não são bem-sucedidos em seus trabalhos estão entre as razões das empresas não admitirem as PCD como candidatos aptos a uma vaga no mercado de trabalho. Além disso, Rocha e Lima (2014) reiteram que ainda existem dificuldades encontradas por empresas para contratarem pessoas com deficiência, pois alegam que as PCD não se adequam ao perfil dessas organizações.

Dessa maneira, cabe a necessidade de entender por completo o que é de fato um mercado de trabalho inclusivo, ou seja, um espaço em que há a participação de todas as pessoas, inclusive, as com deficiência, as vulneráveis, as desfavorecidas, que na visão de alguns podem não produzir o suficiente, porém dão o melhor de si em suas habilidades (EDZES; VAN; RIJNKS, 2013).

Considerando a abrangência e difusão sobre a temática, o presente trabalho tem por objetivo efetuar uma revisão sistemática da literatura sobre a efetiva inclusão de trabalhadores com deficiência, seja no ambiente privado, seja no ambiente público, que cabe destacar as seguintes questões a serem respondidas: que ações, segundo a literatura, têm sido implementadas para promover a visibilidade e empoderamento dos trabalhadores deficientes nos seus espaços de trabalho? O que se tem feito para contribuir com a inclusão dos trabalhadores com deficiência para serem mais bem aproveitados?

O presente trabalho encontra-se organizado em 5 seções, a saber: a introdução, que visa a situação da temática a ser pesquisada. Na segunda seção, encontra-se descrito o método de pesquisa adotado. A terceira seção contempla os resultados obtidos a partir do levantamento da literatura. Na quarta seção, são discutidos os resultados advindos da pesquisa bibliográfica e, finalmente, na quinta e última seção, encontram-se sumarizadas as principais conclusões, além de sugestões de estudos futuros.

\section{Metodologia}

Por se tratar de um estudo que versa sobre a inclusão social das pessoas com deficiência, por meio do mercado de trabalho, utilizou-se, na pesquisa, o protocolo Cochrane, ligado a levantamentos sistemáticos na área da saúde, em virtude da sua robustez e por ser uma área que se correlaciona com o tema a ser pesquisado. Além disso, Higgins, Thomas, Chandler, Cumpston, Li, Page e Welch (2020) orientam que as revisões têm o objetivo de entender as evidências, que as ajuda a tomarem medidas objetivas acerca dos cuidados de saúde. 
Entre os meses de junho e julho de 2019, as bases de dados Scopus (Elsevier), Web of Science (Thomson Reuters Scientific) e SciELO foram consultadas para realizar os levantamentos na literatura científica. Além dessas, com o intuito de evidenciar possíveis obras produzidas nos Programas de Pós-Graduação do Brasil sobre o tema, realizou-se uma pesquisa na Biblioteca Digital Brasileira de Teses e Dissertações (BDTD) do Instituto Brasileiro de Informação em Ciência e Tecnologia (IBICT).

Duas perguntas foram feitas para orientar a revisão sistemática da literatura: que ações institucionais são encontradas na literatura capazes de promover a visibilidade e o empoderamento de pessoas com deficiência nos seus espaços de trabalho? O que as organizações têm realizado com vistas a contribuir para a inclusão ampliada e efetiva de PCD?

Para Heidrich (2016), determinar o foco da pesquisa é, primeiramente, o passo mais importante a ser decidido na elaboração da revisão sistemática. Para tal, é necessário que a questão a ser respondida seja constituída de forma clara. A pergunta de revisão é impulsionada por palavras-chave ou expressões que necessitam ser avaliadas para a sua inserção e busca nas bases científicas de dados escolhidas.

Considerando o protocolo de revisão sistemática adotado, utilizou-se o acrônimo PICO (em inglês: Population, Intervention, Comparison and Outcomes) para apoiar o direcionamento das buscas de palavras-chave/indexadores que se derivam das questões de pesquisa. Dessa forma, para Population acharam-se as palavras-chave: disabled worker, public service; workforce with disability person; physical disability; intellectual or cognitive impairment; public universities.

No caso de Intervention, encontraram-se public policy; inclusion of workers with disabilities; accessibility; professional development; inclusive labor market; diversity. Para Comparison não foi possível encontrar palavras-chave, pois esse atributo, geralmente, é aplicável nas áreas de ciência da saúde.

Por fim, em Outcomes, identificaram-se as palavras-chave valuation; recognition; social inclusion through work; respect; prejudice; social vulnerability; empowerment, visibility; human development; inclusive social development. Assim, foi possível constituir uma string de pesquisa que foi utilizada nas bases de dados expostas acima, adicionando a essa string os conectores boolianos AND e OR. Sendo esses usados para apoiar a grafia da seguinte string de pesquisa avançada:

(("disabled worker in government service" OR "work force with disability person" OR "physical disability" OR "intellectual or cognitive impairment" OR "public universities") AND ("public policy" OR "inclusion of workers with disabilities" OR "accessibility" OR "professional development" OR "inclusive labor market" OR "diversity") AND ("valuation" OR "recognition" OR "social inclusion through work" OR "respect" OR "prejudice" OR "social vulnerability" OR "empowerment" OR "visibility" $O R$ "human development" OR "inclusive social development" ))

Cabe ressaltar que, na Biblioteca Digital Brasileira de Teses e Dissertações (BDTD), utilizaram-se esses mesmos conectores, porém com algumas palavraschave em português, tendo em vista se tratar de um repositório com publicações essencialmente no vernáculo do país.

Inicialmente, após a feitura da pesquisa nas bases de dados com essa string, chegou-se ao resultado total de 5.140 trabalhos, sendo, 108 na Scopus, 2.145 na Web of Science, 215 na SciELO e 2.672 dissertações na BDTD. Para refinar esses 
trabalhos, nas quatro bases de dados, pôde-se aplicar o recurso de filtragem, sendo no total de quatro tipos de filtros, tais como: idioma, tipo de documento, área de estudo e supressão de documentos duplicados na mesma base, conforme sistematizado na Quadro 1.

Quadro 1 - Etapas e critérios para o levantamento sistemático de obras
\begin{tabular}{|l|c|c|c|c|}
\hline \multicolumn{4}{|c|}{ Etap de Seleção } \\
\hline \multicolumn{1}{|c|}{ Base de Pesquisa } & Scopus & SciELO & $\begin{array}{c}\text { Web of } \\
\text { Science }\end{array}$ & BDTD \\
\hline Resultado Inicial & $\mathbf{1 0 8}$ & $\mathbf{2 1 5}$ & $\mathbf{2 . 1 4 5}$ & $\mathbf{2 . 6 7 2}$ \\
\hline $\begin{array}{l}\text { Filtro 1 - Tipo de documento (Artigos, } \\
\text { Revisões e Dissertação/ Teses) }\end{array}$ & 108 & 194 & 1641 & 2.672 \\
\hline Filtro 2 - Área & 108 & 80 & 540 & 161 \\
\hline $\begin{array}{l}\text { Filtro 3 - Idioma (Inglês, Português e } \\
\text { Espanhol) }\end{array}$ & 92 & 80 & 360 & 154 \\
\hline $\begin{array}{l}\text { Filtro 4 - Supressão de documentos } \\
\text { duplicados na mesma base }\end{array}$ & 91 & 72 & 360 & 149 \\
\hline Total de documentos & & \multicolumn{2}{|c|}{672} \\
\hline
\end{tabular}

Fonte: Elaborado pelos autores (2020).

Feita essa análise, partiu-se para a avaliação de uma leitura criteriosa dos resumos, títulos e palavras-chave desses trabalhos, para reconhecer quais que mais se alinhavam à temática dessa pesquisa.

\section{Resultados e discussões}

A presente seção oferece uma sumarização dos achados provenientes dos levantamentos realizados em cada uma das quatro bases científicas previamente mencionadas.

Na Scopus, dos 108 trabalhos identificados inicialmente, após a etapa de filtragem, restaram 92 trabalhos para serem analisados e foi possível identificar um artigo duplicado. Dessa forma, permaneceram 91 trabalhos. A classificação desses foi feita por ano de sua publicação de 1985 até 2019. Dentre esses, mediante leitura aprofundada, apenas 4 artigos foram selecionados, tendo em vista sua aderência ao tema a ser estudado.

Na SciELO, houve uma particularidade na busca pelos trabalhos, isto é, a string de pesquisa foi subdividida em três partes, pois não havia a possibilidade de realizar a busca na sua integralidade. Dos 215 artigos encontrados, 8 estavam duplicados, logo, chegaram-se a 72 artigos finais.Verificou-se que a evolução desses artigos, no que concerne à sua publicação, foi de 2000 a 2019. Contudo, apenas 4 artigos foram selecionados para a revisão da literatura.

$\mathrm{Na}$ Web of Science, foi realizada uma busca avançada redigindo a string de pesquisa sem as aspas, com isso, obtiveram-se 2.145 artigos. Após, aplicaram-se filtros para identificar um resultado mais substancial no que tange aos artigos mais aderentes à temática. Concluída essa etapa, 360 trabalhos foram identificados num espaço temporal de 1995 a 2019, não foi identificada duplicação entre eles e somente 4 trabalhos foram selecionados para a revisão da literatura.

Na Biblioteca Digital Brasileira de Teses e Dissertações (BDTD), apuraram-se os trabalhos distribuídos em 625 teses de doutorados e 2.074 dissertações. Nessa 
base, foram utilizadas algumas palavras da string de pesquisa e redigidas em português, tais como: trabalhador com deficiência no serviço público $O R$ desenvolvimento social inclusivo $O R$ força de trabalho com pessoa com deficiência OR inclusão de trabalhadores com deficiência.

Após o estabelecimento de filtros, havia 154 documentos divididos entre 129 dissertações de mestrado e 25 teses de doutorado. Realizada a leitura dos títulos e resumos destes trabalhos, verificou-se que havia 5 títulos duplicados, o que restaram 149. Dentre esses, mediante uma leitura aprofundada, fez-se a seleção das 7 dissertações que mais se adequaram ao tema proposto.

\subsection{A constituição do núcleo inicial de referências bibliográficas}

Realizada as pesquisas nas bases de dados Scopus, SciELO, Web of Science e Biblioteca Digital Brasileira de Teses e Dissertações, algumas seleções foram realizadas a fim de contribuir na escolha de trabalhos que eram bastante pertinentes ao tema a ser pesquisado.

Mesmo após verificar se havia duplicação de trabalhos na mesma base, foi necessário fazer um cruzamento entre as bases, com o objetivo de verificar a possibilidade de uma nova duplicação, para que houvesse um refinamento neste processo de constituição do núcleo de partida da revisão da literatura. Mediante esse processo, identificaram-se 5 duplicações, restando 667 trabalhos finais para serem examinados. Após uma leitura detalhada dos resumos e títulos desses 667 trabalhos, selecionou-se 18 trabalhos, constando entre eles 7 dissertações de mestrado e 11 artigos científicos.

O Quadro 2 sumariza o método de escolha desses documentos, que se inicia nos resultados antes dos filtros e termina na formação final do núcleo de partida das referências bibliográficas.

\section{Quadro 2 - Formação do Núcleo Inicial das Referências Bibliográficas}

\begin{tabular}{|l|c|c|c|c|}
\hline \multicolumn{1}{|c|}{ Etapas de Seleção } \\
\hline \multicolumn{1}{|c|}{ Base de Pesquisa } & Scopus & SciELO & $\begin{array}{c}\text { Web of } \\
\text { Science }\end{array}$ & BDTD \\
\hline Resultado Inicial & 108 & 215 & 2.145 & 2.672 \\
\hline $\begin{array}{l}\text { Filtro 1 - Tipo de documento (Artigos, } \\
\text { Revisões e Dissertação) }\end{array}$ & 108 & 194 & 1641 & 2.672 \\
\hline Filtro 2 - Área & 108 & 80 & 540 & 161 \\
\hline $\begin{array}{l}\text { Filtro 3 - Idioma (Inglês, Português e } \\
\text { Espanhol) }\end{array}$ & 92 & 80 & 360 & 154 \\
\hline $\begin{array}{l}\text { Filtro 4 - Supressão de documentos } \\
\text { duplicados na mesma base }\end{array}$ & 91 & 72 & 360 & 149 \\
\hline Total de documentos & & 672 \\
\hline $\begin{array}{l}\text { Filtro 5 - Supressão de documentos } \\
\text { duplicados entre as bases }\end{array}$ & \multicolumn{5}{|c|}{667} \\
\hline $\begin{array}{l}\text { Filtro 6 - Seleção baseada em títulos e } \\
\text { resumos }\end{array}$ & \multicolumn{5}{|c|}{18} \\
\hline $\begin{array}{l}\text { Filtro 7 - Documentos selecionados após } \\
\text { etapas anteriores de refinamento }\end{array}$ & \multicolumn{5}{|c|}{} \\
\hline
\end{tabular}

Fonte: Elaborado pelos autores (2020). 
O Quadro 3 oferece a consolidação dos artigos aderentes, seus autores, periódicos e o ano de publicação.

\begin{tabular}{|c|c|c|c|}
\hline Título & Autores & Periódico & $\begin{array}{c}\text { Ano da } \\
\text { Publicação }\end{array}$ \\
\hline $\begin{array}{l}\text { O trabalhador com deficiência e } \\
\text { as práticas de inclusão no } \\
\text { mercado de trabalho de } \\
\text { Salvador, Bahia }\end{array}$ & $\begin{array}{l}\text { Fernando Donato } \\
\text { Vasconcelos }\end{array}$ & $\begin{array}{l}\text { Revista Brasileira de } \\
\text { Saúde Ocupacional }\end{array}$ & 2010 \\
\hline $\begin{array}{l}\text { 'Welcome to my life!' photovoice: } \\
\text { needs assessment of, and by, } \\
\text { persons with physical disabilities } \\
\text { in the kumasi metropolis, ghana }\end{array}$ & $\begin{array}{l}\text { Mandy Tijm; Huib } \\
\text { Cornielje; Anthony } \\
\text { Kwaku Edusei. }\end{array}$ & $\begin{array}{l}\text { Disability, } C B R \quad \& \\
\text { Inclusive } \\
\text { Development }\end{array}$ & 2011 \\
\hline $\begin{array}{l}\text { Employment of Young Adults } \\
\text { With Disabilities in Dubai-A Case } \\
\text { Study: Employment in Dubai }\end{array}$ & $\begin{array}{l}\text { Nadera Alborno; Eman } \\
\text { Gaad. }\end{array}$ & $\begin{array}{l}\text { Journal of Policy and } \\
\text { Practice in } \\
\text { Intellectual } \\
\text { Disabilities }\end{array}$ & 2012 \\
\hline $\begin{array}{l}\text { Trabalhador com deficiência } \\
\text { física: fragilidades e agravos } \\
\text { autorreferidos }\end{array}$ & $\begin{array}{l}\text { Débora Ribas Leal; } \\
\text { Gisele Domingues de } \\
\text { Mattos; Rosane } \\
\text { Teresinha Fontana. } \\
\end{array}$ & $\begin{array}{l}\text { Revista Brasileira de } \\
\text { Enfermagem }\end{array}$ & 2013 \\
\hline $\begin{array}{lcr}\text { Career } & \text { development } & \text { and } \\
\text { individuals } & \text { with } & \text { physical } \\
\text { disabilities } & & \end{array}$ & $\begin{array}{l}\text { Mercedes Villanueva- } \\
\text { Flores; Ramon Valle- } \\
\text { Cabrera; Mar Bornay- } \\
\text { Barrachina }\end{array}$ & $\begin{array}{l}\text { Career Development } \\
\text { International }\end{array}$ & 2014 \\
\hline $\begin{array}{l}\text { Mejores prácticas empresariales } \\
\text { de responsabilidade social en La } \\
\text { inclusión de personas con } \\
\text { discapacidad. Estudio de caso en } \\
\text { empresas de Ambato, Ecuador }\end{array}$ & $\begin{array}{lr}\text { Fanny Paulina } & \text { Pico } \\
\text { Barrionuevo; } & \text { Silvia } \\
\text { Susana Torres. } & \end{array}$ & $\begin{array}{l}\text { Revista de Ciencias } \\
\text { de la Administración } \\
\text { y Economía }\end{array}$ & 2017 \\
\hline $\begin{array}{l}\text { O Trabalho de Colaboradores } \\
\text { com Deficiência nas Empresas: } \\
\text { com a Voz os Gestores de } \\
\text { Recursos Humanos }\end{array}$ & $\begin{array}{lr}\text { Kátia Soares } & \text { Coutinho; } \\
\text { Graciela } & \text { Fagundes } \\
\text { Rodrigues; } & \text { Liliana } \\
\text { Maria Passerino. }\end{array}$ & $\begin{array}{l}\text { Revista Brasileira de } \\
\text { Educação Especial }\end{array}$ & 2017 \\
\hline $\begin{array}{l}\text { Organizações de ensino superior: } \\
\text { inclusão e ambiente de trabalho }\end{array}$ & $\begin{array}{lr}\text { Carlos } & \text { Eduardo } \\
\text { Candido Pereira; José } \\
\text { Luís Bizelli; Lúcia } \\
\text { Pereira Leite. }\end{array}$ & $\begin{array}{l}\text { Educação } \\
\text { Sociedade }\end{array}$ & 2017 \\
\hline $\begin{array}{l}\text { Inclusion Through Work and } \\
\text { Productivity for Persons with } \\
\text { Intellectual and Developmental } \\
\text { Disabilities }\end{array}$ & $\begin{array}{l}\text { Rosemary Lysaght; } \\
\text { Jami Petner-Arrey; } \\
\text { Angela Howell-Moneta; } \\
\text { Virginie Cobigo. }\end{array}$ & $\begin{array}{lr}\text { Journal of } & \text { Applied } \\
\text { Research } & \text { in } \\
\text { Intellectual } & \\
\text { Disabilities } & \\
\end{array}$ & 2017 \\
\hline $\begin{array}{l}\text { Educação inclusiva e as } \\
\text { representações dos estudantes } \\
\text { sobre seus pares com deficiência }\end{array}$ & $\begin{array}{l}\text { Molise de Bem } \\
\text { Magnabosco; Leonardo } \\
\text { Lemos de Souza. }\end{array}$ & $\begin{array}{l}\text { Psicologia Escolar e } \\
\text { Educacional }\end{array}$ & 2018 \\
\hline $\begin{array}{l}\text { Reinserção profissional: } 0 \\
\text { trabalho após a aquisição de uma } \\
\text { deficiência }\end{array}$ & $\begin{array}{ll}\text { Joelma } & \text { Cristina } \\
\text { Santos; Maria Nivalda } \\
\text { de Carvalho-Freitas. }\end{array}$ & $\begin{array}{l}\text { Arquivos Brasileiros } \\
\text { de Psicologia }\end{array}$ & 2018 \\
\hline
\end{tabular}

Fonte: Elaborado pelos autores (2020). 
O Quadro 4 sumariza a relação das dissertações selecionadas na BDTD como também o título, os autores, os programas de Pós-Graduação e o ano de publicação.

Quadro 4 - Relação das dissertações selecionadas na BDTD.

\begin{tabular}{|c|c|c|c|}
\hline Título da Dissertação & Autores & $\begin{array}{c}\text { Programas de Pós- } \\
\text { Graduação }\end{array}$ & $\begin{array}{c}\text { Ano da } \\
\text { Publicação }\end{array}$ \\
\hline $\begin{array}{lll}\text { Professores } & \text { com } & \text { deficiência } \\
\text { física no ensino } & \text { superior: } \\
\text { estudos } & \text { de } & \text { trajetórias } \\
\text { escolares } & & \\
\end{array}$ & $\begin{array}{l}\text { Frederico Kauffann } \\
\text { Barbosa }\end{array}$ & $\begin{array}{l}\text { Faculdades de } \\
\text { Humanidades e Direito - } \\
\text { Universidade Metodista } \\
\text { de São Paulo }\end{array}$ & 2009 \\
\hline $\begin{array}{l}\text { Inclusão de pessoas com } \\
\text { deficiência no mercado de } \\
\text { trabalho: relendo a } \\
\text { desigualdade e a busca da } \\
\text { isonomia }\end{array}$ & Leila Heidrich & $\begin{array}{l}\text { Programa de Pós- } \\
\text { Graduação em Política } \\
\text { Social - Universidade } \\
\text { Católica de Pelotas }\end{array}$ & 2016 \\
\hline $\begin{array}{l}\text { Sentidos e significados do } \\
\text { trabalho para servidores } \\
\text { públicos com deficiência: um } \\
\text { olhar a partir de trajetórias de } \\
\text { vida e trabalho }\end{array}$ & $\begin{array}{l}\text { Amanda Grasiela da } \\
\text { Luz Peres }\end{array}$ & 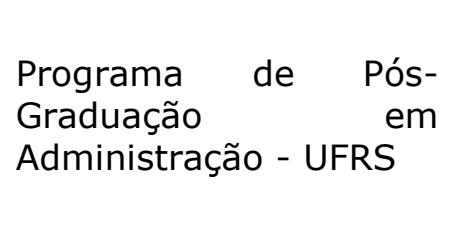 & 2016 \\
\hline $\begin{array}{l}\text { Fluxos de inserção e } \\
\text { acompanhamento das } \\
\text { condições de trabalho de } \\
\text { servidores com deficiência: } \\
\text { um estudo de caso }\end{array}$ & $\begin{array}{l}\text { Pedro Henrique } \\
\text { Fernandes Soares } \\
\text { Costa }\end{array}$ & $\begin{array}{l}\text { Programa de } \quad \text { Pós- } \\
\text { Graduação em Gestão } \\
\text { de } \\
\text { Institucionais - UFRN }\end{array}$ & 2017 \\
\hline $\begin{array}{l}\text { A inclusão de servidores com } \\
\text { deficiência na Universidade } \\
\text { Federal de Juiz de Fora: } \\
\text { diálogos entre princípio da } \\
\text { igualdade, ações afirmativas e } \\
\text { gestão do conhecimento }\end{array}$ & $\begin{array}{l}\text { Thaís Quintão Ferreira } \\
\text { do Vale }\end{array}$ & $\begin{array}{l}\text { Programa de Pós- } \\
\text { Graduação Profissional } \\
\text { em Gestão e Avaliação } \\
\text { da Educação Pública - } \\
\text { UFJF }\end{array}$ & 2017 \\
\hline $\begin{array}{l}\text { Paradoxos da inclusão no } \\
\text { trabalho: um estudo com } \\
\text { pessoas com deficiência no } \\
\text { setor público }\end{array}$ & Davi Sampaio Marques & $\begin{array}{lr}\text { Programa de } & \text { Pós- } \\
\text { Graduação } & \text { em } \\
\text { Administração } & \text { e } \\
\text { Controladoria - UFCE }\end{array}$ & 2018 \\
\hline $\begin{array}{l}\text { Gestão Inclusiva: Propostas } \\
\text { para a Inclusão Laboral em } \\
\text { uma IFES }\end{array}$ & José Luis Machado & $\begin{array}{lr}\text { Programa de } & \text { Pós- } \\
\text { Graduação } & \text { em } \\
\text { Engenharia de Produção } \\
\text { - UFRS }\end{array}$ & 2019 \\
\hline
\end{tabular}

Fonte: Elaborado pelos autores (2020).

Os resultados do levantamento sistemático da literatura permitiram a identificação de diversos assuntos que estavam ligados à inclusão, como: deficiência; mercado de trabalho inclusivo para as PCD, e; servidor público deficiente na universidade pública.

\subsection{A contextualização histórica sobre deficiência}

Relevante assinalar que, segundo relata Heidrich (2016), a expressão deficiência fora identificada na literatura com variados significados, muitas vezes 
preconceituosos, que se relacionavam com as pessoas que nasciam com a deficiência ou até mesmo as adquiriam em acidentes ou doenças, tais como pessoas incapacitadas, defeituosas, pessoas portadoras de deficiência, pessoas com necessidades especiais entre outros foram dados às PCD no decorrer dos anos. As expressões depreciativas relacionadas às PCD somente davam-Ihes a função de descrédito junto à sociedade (BARBOSA, 2009).

Saliente-se ainda que, o modo de como a sociedade se relaciona com as pessoas com deficiência reflete a história de exclusão desses indivíduos, que eram segregados, pelo fato de serem vistos como pessoas incapazes os quais ficavam restritos a instituições especializadas para tal fim (MAGNABOSCO; SOUZA, 2018).

\subsection{A inclusão da pessoa com deficiência}

Ao abordar sobre a inclusão das pessoas com deficiência, é perceptível que a sua inserção na vida social, econômica e política é uma maneira de respeitar os seus direitos no que abrange à Sociedade, ao Estado e ao Poder Público conforme estudo realizado por Heidrich (2016). Ao corroborar com a ideia de Heidrich (2016), a inclusão, de acordo com Pereira, Bizelli e Leite (2017), é um exercício que deve ser praticado dia a dia para se vencer os preconceitos que são praticados pela sociedade, mesmo que o poder governamental venha reprimir, por meio de suas ações, o desrespeito às diferenças.

Sobre o processo de inclusão das pessoas com deficiências em outros países, Alborno e Gaad (2012) trazem, em sua pesquisa, o conhecimento da criação de uma lei nos Estados Unidos da América (EUA), a chamada American with Disability Act (ADA), que tem a finalidade de preservar todos os direitos das PCD nesse país. A ADA os ajuda não somente a dar acesso às oportunidades de empregos como também a combater a discriminação nos seus ambientes de trabalho. Além de promulgarem uma norma que amparam as PCD, os EUA também fiscalizam as empresas que empregam as PCD, no intuito de verificar se essas cumprem o seu papel de empregadores no que tange aos processos de recrutamento, condições de trabalho e remuneração.

\subsection{Preconceitos e visões distorcidas sobre as PCD}

As barreiras físicas como edifícios públicos e meios de transportes inacessíveis não são as únicas desvantagens que as pessoas com deficiência físicas sofrem, há outros tipos de desvantagens que podem estar presentes na vida dessas pessoas como as barreiras sociais. Atitudes negativas impostas pela sociedade como estigma e negligência são as que essas pessoas com deficiência têm de lidar. Ainda que os seus direitos estejam amparados por lei, essas pessoas vivem, muitas das vezes, excluídas de posições de liderança como também da sua participação em discussões públicas e políticas (TIJM; CORNIELJE; EDUSEI, 2011).

Nesse contexto, Vasconcelos (2010) alega que a sociedade ainda não está apta a lidar com as diferenças, sejam elas corporais, de habilidades ou de classes, assim, ao se criar quaisquer políticas públicas que envolvam as pessoas com deficiência, essas inaptidões da sociedade devem ser revistas.

Como observado por Peres (2016), o preconceito é exposto pelos demais através dos estereótipos que são atrelados às PCD, o que gera características negativas para estes indivíduos, além da desvalorização, da desconfiança e da criação de barreiras que possam impossibilitar de terem acesso aos bens sociais. Nesse 
sentido, o prejulgamento pode influenciar em atitudes e comportamentos que prejudicam as PCD em oportunidades de acesso ao trabalho, de uma formação educacional ou até mesmo de uma qualificação.

\subsection{A inserção das PCD no mercado de trabalho}

A inserção no ambiente laboral pela via formal, além de ser uma das maneiras de se ter alguma renda, provoca nas pessoas a aquisição de características que podem identificá-las. Ter uma vida profissional confirma o lugar dessa pessoa na sociedade, ajuda-a determinar quem são as pessoas que fazem parte do seu vínculo afetivo e construir competências, o que colabora para que ela tenha saúde mental e física (SANTOS; CARVALHO-FREITAS, 2018).

Coutinho, Rodrigues e Passerino (2017) afirmam ser a inserção das pessoas com deficiência no mercado de trabalho uma maneira delas verem a sua cidadania conquistada e exercida, assim, conclui-se que a sua inclusão as tornam capazes de perceber o quanto podem produzir para a sociedade, logo, estigmas e pensamentos preconceituosos enraizados podem ser reduzidos. Para esses autores, as dificuldades encontradas pelas $P C D$ em relação a não capacitação e as dificuldades de conquistarem seus espaços nos ambientes laborais podem ser diminuídas a partir da elaboração de políticas que venham para reforçar a sua inclusão nas empresas, além de práticas que ratifiquem a sua educação.

Além disso, Coutinho, Rodrigues e Passerino (2017) ratificam que se não forem formuladas estratégias para tornarem acessíveis ações na área educacional, nos postos de trabalhos e no dia a dia das PCD, haverá a possibilidade de barreiras que poderão limitar o seu conhecimento e a sua aprendizagem. As pessoas com deficiência quando não realizam as suas atividades nos seus locais de trabalho, devido às suas limitações, são vistas com olhares de rejeição pelos demais, que as culpam por essas limitações que provêm da sua própria deficiência. Um dos principais fundamentos de qualquer organização empregadora é a garantia de tratamento justo dos empregados no local de trabalho (VILLANUEVA-FLORES; VALLE-CABRERA; BORNAY-BARRACHINA, 2014).

Vasconcelos (2010) evidencia que as vagas ofertadas às pessoas com deficiência pela política de cotas, além de uma obrigação, é uma forma de que as empresas possam estar sensíveis na questão da inclusão dessas pessoas nos ambientes empresariais. A finalidade dessa reserva de vagas, por meio desta política, é apontar que as PCD possuem capacidades e competências, o que auxilia na mudança da ideia formada que a sociedade tem como improdutividade, incapacidade e dependência.

Em outro momento, a história mostrou que as PCD eram desacreditadas, no que abrange ao seu talento, porém, já há mudanças nesse perfil que outrora era de improdutividade. A Lei de Cotas, que se encontra em evolução, traz para as PCD a sua empregabilidade, é o que destaca Coutinho, Rodrigues e Passerino (2017).

Nesse enquadramento, Marques (2018) reforça a ideia de que um dos grandes desafios a ser vencido é a efetiva inclusão das $P C D$, principalmente, no mercado de trabalho. As empresas além de imporem algumas barreiras, dificultam a entrada e permanência dessas pessoas nos espaços empresariais, mesmo que a Lei de Cotas os imponha de contratar, sendo poucas as chances ofertadas às PCD, o que de fato não deve ocorrer. 
Uma empresa é inclusiva quando transmite uma cultura organizacional que fomenta a empatia entre seus colaboradores, ou seja, as pessoas em suas relações usam palavras e gestos que possam acarretar afeto pelas outras pessoas, assim essa cultura deve ser preservada e cuidada, pois, é preciso que o desenvolvimento dessa cultura não aconteça somente pelos atos, mas sim, deve-se criar uma formalidade constituída nos processos e métodos de ação dessa instituição (MACHADO, 2019).

Além do mais, para Leal, Mattos e Fontana (2013), há ainda um grande avanço a ser conquistado quando se fala em inclusão eficaz das PCD no mercado de trabalho, mesmo já tendo uma lei que, desde da década de 90, vem a fomentar a inclusão laboral desses indivíduos.

Barrionuevo e Torres (2017) demonstram que as pessoas com algum tipo de deficiência mais significativa são as mais prejudicadas, sendo as que possuem uma menor participação no mercado de trabalho. É preciso pensar na existência de lacunas na inclusão das PCD, as quais devem ser preenchidas e podem tornar essas pessoas mais preparadas, pois esses indivíduos já sentiram a exclusão do mercado de trabalho de formas variadas. O setor empresarial tem, em seu poder, a chance, seja por meio do cumprimento de normais legais ou por responsabilidade social, de incluir verdadeiramente as PCD na sociedade, através do trabalho.

Dessa forma, Lysaght et al. (2017) dizem que socializar para as pessoas com deficiência é uma das causas que os mais motivam quando estão em um ambiente de trabalho, permanecer no trabalho é muito importante e os ajudam no reconhecimento e na sua aceitação por parte de outras pessoas.

\subsection{O trabalhador com deficiência no Serviço Público}

Ao tratar sobre inclusão das pessoas com deficiência na Administração Pública brasileira, como servidores públicos, tem-se então uma lei de suma importância (Lei no 8.112/1990) que trouxe para o ordenamento jurídico a inserção dessas pessoas, por meio de concurso público, para atuar nas repartições públicas, através da reserva de vagas (MACHADO, 2019). Esse dispositivo informa que a Administração pública deverá reservar de cinco a vinte por cento das vagas que são oferecidas em concursos públicos para as pessoas com deficiência (COSTA; OLIVEIRA, 2017).

Na opinião de Peres (2016), o trabalho desenvolvido pelos servidores com deficiência nos órgãos públicos tem relevância e estes indivíduos percebem que no seu desempenho há um grande significado, porque mesmo que ainda sofram algum tipo de discriminação, possuem um grau maior de independência e podem sentir-se mais reconhecidos no contexto do trabalho.

Para que esse grupo de pessoas conquiste uma maior autonomia é necessário discutir outro ponto importante: a acessibilidade, pois é um dos elementos fundamentais para a vida das PCD. Os gestores que administram as instituições públicas precisam compreender e seguir essa linha de pensamento, dessa forma verão a dimensão que a acessibilidade causa para os servidores com deficiência, além de proporcionar a efetiva inserção nos espaços de trabalho das PCD (COSTA; OLIVEIRA, 2017).

Costa e Oliveira (2017), a respeito da acessibilidade, em relação a Peres (2016), dizem ser o trabalho adaptado condição básica para que o servidor com deficiência exerça as suas funções com autonomia, o que nem sempre acontece. Ainda não há uma preocupação das instituições com essas questões. 
Quando os servidores com deficiência entram numa instituição pública é preciso perceber outras questões relevantes para que esses possam obter crescimento em condições iguais a de outros servidores, conforme salienta Valle (2017). Além disso, para Valle (2017) é necessário que capacitem os servidores com deficiência, de forma que a instituição forneça recursos adequados e acessíveis, o que implica nisto a sua devida alocação no ambiente laboral, além de considerar a sua mobilidade dentro do seu espaço de trabalho como também o perfil desses servidores e as funções que eles desempenharão no seu dia a dia.

Assim, ao fundamentar o entendimento de Valle (2017), Peres (2016) afirma que no âmbito de reconhecer e valorizar, qualidades como competência e habilidade na execução do trabalho dos servidores deficientes, faz com que o rótulo de incapacidade das PCD seja desmitificado, dessa forma, traz para o cotidiano uma menor percepção das suas limitações. O trabalho para esses indivíduos é uma conquista, seja financeira, seja pessoal, além de trazer-lhes a sensação de poder fazer parte de um grupo e de se relacionarem com outras pessoas. De fato, as PCD quando contribuem para a sociedade, por meio do serviço público, colaborarão para o bem comum.

A Figura 1 apresenta a sistematização da promoção de ações que possam ser promovidas dentro dos ambientes de trabalho das pessoas com deficiência.

Figura 1: Boas práticas promovidas por organizações inclusivas

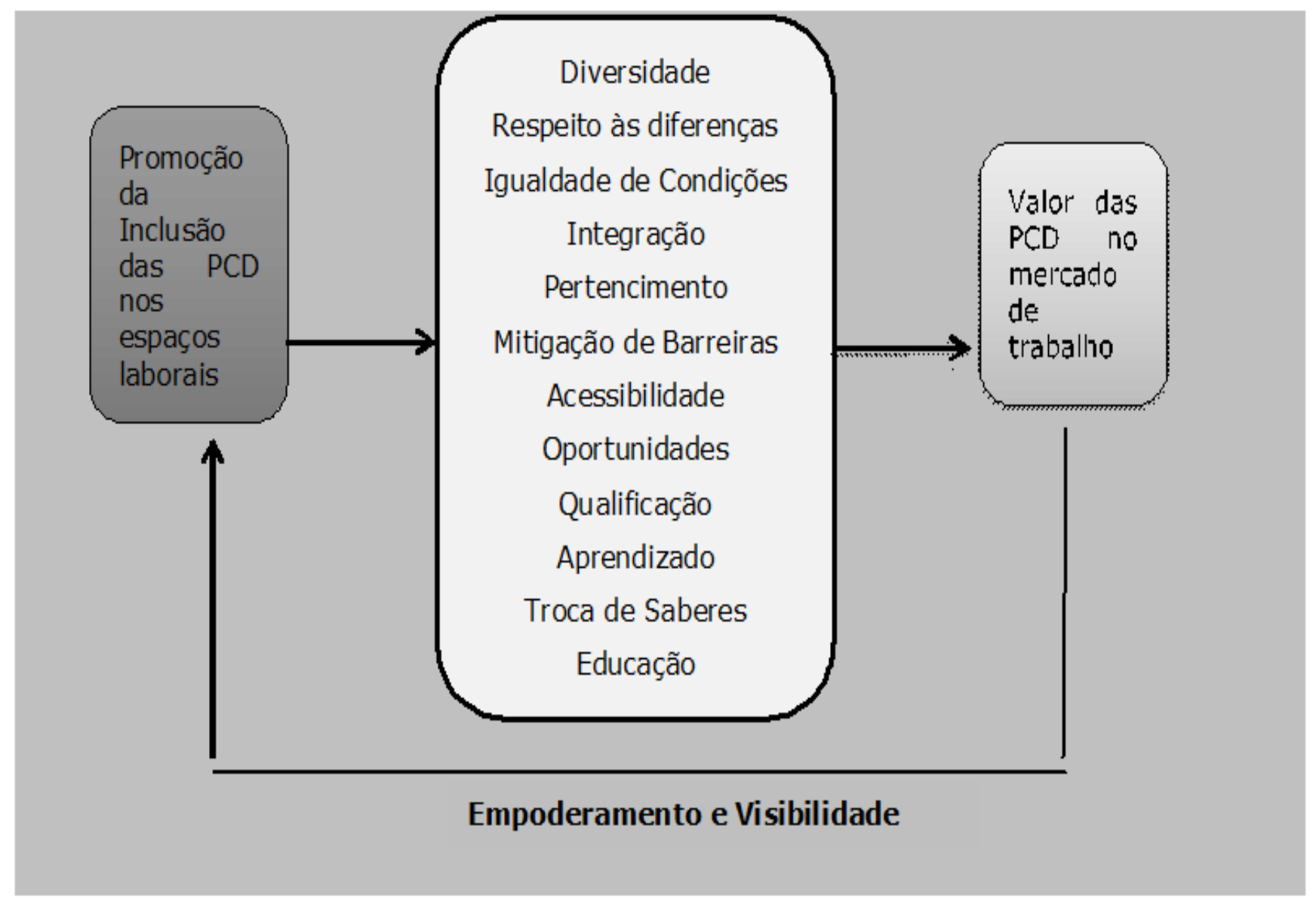

Fonte: Elaborado pelos autores (2020). 
Como é possível observar, o input sinalizado no fluxograma da Figura 1, das boas práticas promovidas pelas organizações inclusivas, dar-se-á com a promoção da inclusão das PCD nos espaços laborais, que resultará num processo de ações que serão desenvolvidas junto às PCD em seus postos de trabalho, segundo afirmam os teóricos da revisão da literatura desta pesquisa. O fomento da prática dessas ações possibilitará verificar o output desse processo, que é o valor desses indivíduos no mercado de trabalho, tendo, ainda, como feedback o empoderamento e a visibilidade das pessoas com deficiência.

\section{Considerações finais}

Conforme os achados teóricos realizados nesta pesquisa, as práticas de uma inclusão mais efetiva são destacadas na revisão da literatura, sendo clara a visão de que deverá haver sérias mudanças para que isso ocorra na prática. Assuntos como preconceitos e estigmas são notórios, principalmente, quando se fala em preconceito velado por parte da sociedade em relação às pessoas com deficiência. A inclusão desses indivíduos nos espaços da sociedade é algo que deve ser disseminado e bastante trabalhado em todos os tipos de ambientes, esferas e classes sociais. Contudo, mesmo que a comunidade científica promova a diversidade, mudar ações e atitudes constituídas é certamente muito complexo, ainda mais quando estão enraizadas na cultura da sociedade a ponto de bloquear sua visão de pensar no novo e enxergar o ser humano que está à sua frente na sua completude.

É necessário à sociedade descortinar o diferente, entender que as pessoas com deficiência também são seres humanos iguais aos outros e merecem ter a chance de mostrar as suas habilidades, e não mais serem marginalizadas.

"A inclusão social é percebida pela participação das pessoas com deficiências na população economicamente ativa" (SOUZA; FRANÇOZO; KASSAR, 2018, p. 221). O sentido da inclusão, através do trabalho, possibilitará a estas pessoas oportunidade de obter novos conhecimentos e desenvolver seu intelecto, não se esquecendo de suas limitações. Porém, isso hoje não é obstáculo para tais conquistas, além do mais, cada vez mais se devem divulgar relatos de superações a fim de que a sociedade compreenda e aceite o potencial que estão nas PCD. Nota-se que a falta de informação da sociedade em relação às possibilidades das PCD dificulta um pouco essa interseção entre as pessoas.

Ao responder as questões da pesquisa, a literatura encontrada na revisão traz as ações tais como a inserção e a empregabilidade das pessoas com deficiência nos ambientes de trabalho como forma de promover a sua visibilidade para a sociedade e também traz para essas pessoas a sensação de pertencimento e interação com outros grupos dos quais já estão habituados a lidarem. Ações que fomentem o entretenimento, a cultura e, principalmente, a educação são práticas de responsabilidade social promovidas pelo Estado e pela sociedade para que possam dar amplo empoderamento às PCD.

Evidencia-se no presente estudo que as empresas, devido a exigência da legislação, contratam em seus espaços, as PCD, e com isto abre a oportunidade e a esperança dessas pessoas de se relacionarem, de serem reconhecidas pelo seu trabalho, de terem a possibilidade de superar obstáculos, e, principalmente a de sonhar com uma vida com mais qualidade. 
Como sugestões de estudos futuros propõem-se pesquisas que possam investigar o comportamento das pessoas com deficiência em relação a estar empregado e como essa ação afeta o seu bem-estar e sua qualidade de vida, além do desenvolvimento adequado das suas tarefas dentro de seus espaços laborais.

\section{REFERÊNCIAS}

ALBORNO, Nadera; GAAD, Eman. Employment of Young Adults With Disabilities in Dubai - A Case Study. Journal of Policy and Practice in Intellectual

Disabilities. Austrália, v. 9, n. 2, p. 103-111, jun. 2012. Disponível em: https://onlinelibrary.wiley.com/doi/pdf/10.1111/j.1741-

1130.2012.00341.x?casa_token=k3PeohVFdJ0AAAAA:xRQsA6E6qroRSkt08MiZoDtN Po3ZCdk7h6qpk-Wb6IF4ag0-6WohdrZm0SJWIGzpGdvFG0Bu_cAKyKo. Acesso em: jun. 2019.

BARBOSA, Frederico Kauffmann. Professores com deficiência física no ensino superior: estudo de trajetórias escolares. 2009. 95 f. Dissertação (Mestrado em Educação) - Universidade Metodista de São Paulo, São Paulo, 2009.

BARRIONUEVO, Fanny Paulina Pico; Torres, Silvia Susana. Mejores prácticas empresariales de responsabilidad social en la inclusión de personas com discapacidad. Estudio de caso en empresas de Ambato, Ecuador. Revista de Ciencias de la Administración y Economía. Equador, v. 14, n. 8, p. 189-200, mar. 2017. Disponível em:

http://scielo.senescyt.gob.ec/scielo.php?script=sci_arttext\&pid=S139086182017000200189. Acesso em: jun. 2019.

BRASIL. Decreto no 3.298, de 20 de dezembro de 1999. Regulamenta a Lei no 7.853 , de 24 de outubro de 1989, dispõe sobre a política nacional para a integração da pessoa portadora de deficiência, consolida as normas e dá outras providências. Diário Oficial da União, Brasília, DF, 21 dez. 1999. Disponível em:

http://www.planalto.gov.br/ccivil_03/decreto/d3298.htm .Acesso em: jul. 2019.

BRASIL. Congresso Nacional. Lei no 13.146, de 6 de julho de 2015 . Institui a Lei Brasileira de Inclusão da Pessoa com Deficiência (Estatuto da Pessoa com Deficiência). Diário Oficial da União, Brasília, DF, 7 jul. 2015. Disponível em: http://www.planalto.gov.br/ccivil_03/_ato2015-2018/2015/lei/l13146.htm. Acesso em: jul. 2019.

COSTA, Larissa Aparecida; OLIVEIRA, Lourival José de. Valorização do trabalho humano e inclusão social no Brasil: a responsabilidade empresarial e as políticas públicas para a inclusão das pessoas com deficiência. Revista de Direito do Trabalho e Meio Ambiente do Trabalho. Brasília, v. 3, n. 1, p. 76-93, jun. 2017. Disponível em:

https://www.researchgate.net/publication/322679578_VALORIZACAO_DO_TRABAL HO_HUMANO_E_INCLUSAO_SOCIAL_NO_BRASIL_A_RESPONSABILIDADE_EMPRES 
ARIAL_E_AS_POLITICAS_PUBLICAS_PARA_A_INCLUSAO_DAS_PESSOAS_COM_DEF ICIENCIA. Acesso em: jun. 2019.

COUTINHO, Kátia Soares; RODRIGUES, Graciela Fagundes; PASSERINO, Liliana Maria. O trabalho de colaboradores com deficiência nas empresas: com a voz os gestores de recursos humanos. Revista Brasileira de Educação Especial. Marília, v. 23, n. 2, p. 261-278, jun. 2017. Disponível em: https://www.scielo.br/scielo.php?pid=S1413$65382017000200261 \&$ script=sci_arttext. Acesso em: jun. 2019.

EDZES, Arjen J.E.; RIJNKS, Richard; DIJK, Jouke van. Spatial Implications of Using Firm Level Quotas to Employ Low Productive Workers. Tijdschrift voor

Economische en Sociale Geografie. Países Baixos, v. 104, n. 5, p. 621-629, set. 2013. Disponível em:

https://onlinelibrary.wiley.com/doi/pdf/10.1111/tesg.12060?casa_token=k0IdloNX oCwAAAAA:AhvbAa4hJrl4FqRsfHydCB96pQUxvUvua_MQeeujtHwUHAIKbF4jV0XidB Cuy3ASQzhXNF8cFxzAwA. Acesso em: jun. 2019.

HEIDRICH, Leila. Inclusão de pessoas com deficiência no mercado de trabalho: relendo a desigualdade e a busca da isonomia. 2016. $113 \mathrm{f}$. Dissertação (Mestrado em Política Social) - Universidade Católica de Pelotas, Pelotas, Rio Grande do Sul, 2016.

HIGGINS, Julian; THOMAS, James; CHANDLER, Jacqueline; CUMPSTON, Miranda; LI, Tianjing; PAGE, Matthew; WELCH, Vivian. Manual Cochrane para revisões sistemáticas de intervenções versão 6.1 (atualizado em setembro de 2020). Cochrane, 2020. Disponível em: https://training.cochrane.org/cochrane-handbooksystematic-reviews-interventions. Acesso em: jun. 2019.

KUZNETSOVA, Yuliya; BENTO, João Paulo Cerdeira. Workplace Adaptations Promoting the Inclusion of Persons with Disabilities in Mainstream Employment: $A$ Case-Study on Employers' Responses in Norway. Social Inclusion. Noruega, v. 6, n. 2, p. 34-45, maio, 2018. Disponível em:

https://www.ssoar.info/ssoar/bitstream/handle/document/57370/ssoarsocialinclusion-2018-2-bento_et_al-

Workplace_adaptations_promoting_the_inclusion.pdf?sequence $=1$ \&isAllowed $=y \& \ln k$ name=ssoar-socialinclusion-2018-2-bento_et_al-

Workplace_adaptations_promoting_the_inclusion.pdf. Acesso em: jun. 2019.

LEAL, Débora Ribas; MATTOS, Gisele Domingues de; FONTANA, Rosane Teresinha. Trabalhador com deficiência física: fragilidades e agravos autorreferidos. Revista Brasileira de Enfermagem. Brasília, v. 66, n. 1, p. 59-66, fev. 2013. Disponível em: https://www.redalyc.org/pdf/2670/267028450009.pdf. Acesso em: jun. 2019.

LYSAGHT, Rosemary; PETNER-ARREY, Jami; HOWELL-MONETA, Angela; COBIGO, Virginie. Inclusion through work and productivity for persons with intellectual and developmental disabilities. Journal of Applied Research in Intellectual 
Disabilities. Canadá, v. 30, n. 5, p. 922-935, ago. 2017. Disponível em:

https://onlinelibrary.wiley.com/doi/epdf/10.1111/jar.12284. Acesso em: jun. 2019.

MACHADO, José Luis. Gestão inclusiva: propostas para a inclusão laboral em uma IFES. 2019. 166 f. Dissertação (Mestrado em Engenharia de Produção) Universidade Federal do Rio Grande do Sul, Porto Alegre, 2019.

MAGNABOSCO, Molise de Bem; SOUZA, Leonardo Lemos de. Educação inclusiva e as representações dos estudantes sobre seus pares com deficiência. Psicol. Esc. Educ. Maringá, v. 22, n. 1, p. 115-122, abr. 2018. Disponível em:

https://www.scielo.br/scielo.php?pid=S1413-

85572018000100115\&script=sci_abstract\&tIng=es. Acesso em: jun. 2019.

MARQUES, Davi Sampaio. Paradoxos da inclusão no trabalho: um estudo com pessoas com deficiência no setor público. 2018. 96f. Dissertação (Mestrado em Administração e Controladoria) - Universidade Federal do Ceará, Fortaleza 2018.

NEVES-SILVA, Priscila; PRAIS, Fabiana Gomes; SILVEIRA, Andréa Maria. Inclusão da pessoa com deficiência no mercado de trabalho em Belo Horizonte, Brasil: cenário e perspectiva. Ciências em Saúde Coletiva. Rio de Janeiro, v. 20, n. 8, p. 2549-2558, ago. 2015. Disponível em:

https://www.scielo.br/scielo.php?pid=S1413-

81232015000802549\&script=sci_abstract\&tlng=pt. Acesso em: jun. 2019.

PEREIRA, Carlos Eduardo Candido; BIZELLI, José Luís; LEITE, Lúcia Pereira. Organizações de ensino superior: inclusão e ambiente de trabalho. Educação \& Sociedade. Campinas, v. 38, n. 138, p. 99-115, jan. 2017. Disponível em: https://www.scielo.br/scielo.php?pid=S0101-

73302017000100099\&script=sci_abstract\&tlng=pt. Acesso em: jul. 2019.

PERES, Amanda Grasiela da Luz. Sentidos e significados do trabalho para servidores públicos com deficiência: um olhar a partir de trajetórias de vida e trabalho. 2016. 182 f. Dissertação (Mestrado em Administração) Universidade Federal do Rio Grande do Sul, Porto Alegre, 2016.

ROCHA, Patrícia da Conceição; LIMA, Iana Bezerra. Inclusão de deficientes físicos no mercado de trabalho: estudo de caso em uma organização no segmento industrial. Revista Eletrônica Interdisciplinar em Negócios e Hospitalidade REINH. v. 2, n. 1, p. 74-93, 2014.

SANTOS, Joelma Cristina; CARVALHO-FREITAS, Maria Nivalda de. Reinserção profissional: o trabalho após a aquisição de uma deficiência. Arquivos Brasileiros de Psicologia. Rio de Janeiro, v. 70, n. 3, p. 184-197, 2018. Disponível em: http://pepsic.bvsalud.org/scielo.php?pid=S1809-

52672018000300013\&script=sci_abstract\&tlng=es. Acesso em: jun. 2019.

SASSAKI, Romeu Kazumi. Inclusão - Construindo uma sociedade para todos. $7^{a}$ ed. Rio de Janeiro: WVA, 2010. 
SOUZA-SILVA, João Roberto de; DIEGUES, Débora; CARVALHO, Sueli Galego de.

Trabalho e deficiência: Reflexões sobre as dificuldades da inclusão social.

Cadernos de Pós-Graduação em Distúrbios do Desenvolvimento, São Paulo, v. 12, n. 1, p. 27-33, 2012. Disponível em:

http://editorarevistas.mackenzie.br/index.php/cpgdd/article/view/11189/6947.

Acesso em: jun. 2019.

SOUZA, Márcia Paula de; FRANÇOZO, Rafael Verão; KASSAR, Mônica de Carvalho Magalhães. Acesso ao Trabalho às Pessoas com Deficiência: Notas sobre o Ingresso à carreira do Funcionalismo Federal. Perspectivas em Diálogo: revista de educação e sociedade. Naviraí, v. 5, n. 9, p. 217-233, jun. 2018. Disponível em: https://periodicos.ufms.br/index.php/persdia/article/view/5181. Acesso em: fev. 2020.

TIJM, Mandy; CORNIELJE, Huib; KWAKU, Anthony Edusei. 'Welcome to my life!' Photovoice: Needs assessment of, and by, persons with physical disabilities in the Kumasi Metropolis, Ghana. Disability, CBR \& Inclusive Development. Gana, v. 22, n. 1, p. 55-72, maio, 2011. Disponível em:

https://www.researchgate.net/publication/265058038_'Welcome_to_my_life'_phot ovoice_Needs_assessment_of_and_by_persons_with_physical_disabilities_in_the_k umasi_metropolis_Ghana Acesso em: jun. 2019.

VALLE, Thais Quintão Ferreira do. A inclusão de servidores com deficiência na Universidade Federal de Juiz de Fora: diálogos entre princípio da igualdade, ações afirmativas e gestão do conhecimento. 2017. $144 \mathrm{f}$. Dissertação (Mestrado Profissional em Gestão e Avaliação da Educação Pública). Universidade Federal de Juiz de Fora, Minas Gerais, 2017.

VASCONCELOS, Fernando Donato. O trabalhador com deficiência e as práticas de inclusão no mercado de trabalho de Salvador, Bahia. Revista Brasileira de Saúde Ocupacional. São Paulo, v. 35, n. 121, p. 41-52, jun. 2010. Disponível em: https://www.scielo.br/scielo.php?script=sci_arttext\&pid=S030376572010000100006 . Acesso em: jun. 2019.

VASH, Carolyn. Enfrentando a deficiência: a manifestação, a psicologia, a reabilitação. São Paulo: Pioneira/EDUSP, 1988.

VILLANUEVA-FLORES, Mercedes; VALLE-CABRERA, Ramón; BORNAY-BARRACHINA, Mar. Career development and individuals with physical disabilities. Career Development International, Austrália, v. 19, n. 2, p. 222-243, mar. 2014. Disponível em: https://www.emerald.com/insight/content/doi/10.1108/CDI-022013-0022/full/pdf?title=career-development-and-individuals-with-physicaldisabilities. Acesso em: jun. 2019.

VORNHOLT, Katharina; VILLOTTI, Patrizia; MUSCHALLA, Beate; BAUER, Jana; COLELLA, Adrienne; ZIJLSTRA, Fred; RUITENBEEK, Gemma Van; UITDEWILLIGEN, Sjir; CORBIĖRE, Marc. Disability and employment - overview and 
highlights. European Journal of Work and Organizational Psychology.

Inglaterra, v. 27, n.1, p. 40-55, out. 2018. Disponível em:

https://www.tandfonline.com/doi/pdf/10.1080/1359432X.2017.1387536?needAcce ss=true. Acesso em: jun. 2019.

Recebido em: 25 de maio de 2020. Aceito em: 29 de novembro de 2020. Publicado em: 05 de janeiro de 2021. 